\title{
o-10. Spinal Cord Compression Study (First Report)
}

\author{
Hiroaki FUKUDA \\ Department of Orthopedic Surgery School of Medicine, Keio University
}

The pathogenesis of compression myelitis has been a subject of long-standing dispute. The contention of circulatory disturbance in the spinal cord, as a causal factor seems to have been supported by many investigators. On the other hand, Tarlov recently advocated that mechanical stress played an important role in producing paralysis at least in acute cases.

This study was therefore undertaken to attempt to forecast its prognosis as wel as to carify its pathogenesis.

Using 51 mongre dogs, 9 reversible spinal paralyses were produced by introducing into the extradural space, a piece of dried laminaria or a balloon which was later inflated. The paralyzed dogs were decompressed at different times and the motor function and pain appreciation were observed periodically.

The microangiogram of spinal artery in the 9 recovered dogs after decompression revealed patent or slightly deviated anterior spinal artery. Even the paraplegic dogs showed almost the same pattern. The venous microangiogram in one dog which had been partially decompressed and returned to almost normal function revealed remarkable defect at the compressed site.

The histologic changes in this group were generally slight in extent. However, a few huge cavitations were observed in the dogs whose clinical course had been uneventful. The location of these cavitations did not seemingly correspond with the arterial distribution. In the recovery process of motor function the length of time until unstable standing and walking roughly paralleled the length of time of compression. As far a sreversibility is concerned, the spinal cord resisted longer period in the relatively gradual compression by the laminaria than in the caute compression by the balloon.

With the relatively acute compression myelitis, mechanical stress appears to be of primary concernment, and circulatory disturbance secondary.

\section{o-11. A long follow-up Study of 128 Cases of Intramedullary Spinal Cord Tumor}

\author{
Hiroo CHIGASAKI \\ Dept. of Neurosurg., Univ. of Tokyo \\ Joe Pennybacker \\ Dept. of Neurological Surgery, Radcliffe Infirmary, Oxford
}

